\title{
Comparative study of herbal compounds curcumin and negundoside on the biofilm producing property of streptococcus mutans isolated from oral infection
}

\begin{abstract}
Biofilm is a community of cells attached to either a biotic or abiotic surface enclosed in a complex exopolymeric substance (EPS). Biofilms allow micro-organisms to trap nutrients and withstand hostile environmental conditions by Quorum sensing (QS). Several serious infections and disease are reported as a result of biofilm. This study aimed to evaluate the efficacy of natural compounds Curcumin and Negundoside on the biofilm producing property of Streptococcus mutans. Samples were collected from patients having oral infection and 30 isolates were identified as S. mutans and screened for biofilm formation by using microtiter plate method. Strongest biofilm producer SM03 was used for minimum inhibitory concentration and minimum biofilm inhibitory concentration. Subsequently this concentration used against each of strong biofilm producer isolates at $492<0.5$ optical density (OD). The 30 isolates screened for biofilm formation, 18 isolates showed strong biofilm formation, 09 isolates showed moderate formation and 03 isolates showed poor/non biofilm formation. The MIC of Curcumin for the strongest biofilm producer SM03 was, $0.63 \pm 0.02$ whereas that of Negundoside was $0.48 \pm 0.02$ and minimum biofilm inhibition concentration of Curcumin was $0.0570 \pm 0.03$ and Negundoside $0.0417 \pm 0.03$ which was lower than the Curcumin. The MBIC of both compounds significantly inhibited biofilm formation of all the 18 strong biofilm-forming isolates. The results of this study demonstrated significant antibiofilm effect of the natural compound Curcumin and Negundoside which contribute towards the development of database for novel drug candidates for treating oral infection caused by $S$. mutans.
\end{abstract}

Keywords: S. mutans, biofilm, natrual compound, curcumin, negundoside
Volume I 3 Issue I - 2020

\author{
Deepak Dwivedi,' Nivedita Vats, ${ }^{2}$ Sourabh \\ Malviya' \\ 'Minor Forest Produce Processing and Research Center, India \\ ${ }^{2}$ Department of Microbiology, Career College Bhopal, India
}

Correspondence: Deepak Dwivedi, Minor Forest Produce Processing and Research Center Barkheda Pathani, Bhopal, Pincode-46202I, M.P., India, Tel no: +91755-2417670; +919893088024, Email microbio.dee@gmail.com

Received: November 25, 2019 | Published: January 09, 2020
Abbreviations: EPS, exopolymeric substance; QS, quorum sensing; AI, autoinducers; BHI, brain heart infusion; MSA, mutant sanguis agar

\section{Introduction}

Biofilm is a community of cells attached to either a biotic or abiotic surface enclosed in a complex exopolymeric substance (EPS). ${ }^{1}$ Biofilms allow micro-organisms to trap nutrients and withstand hostile environmental conditions by Quorum sensing (QS). It is widespread and well-known cell-to-cell communication phenomenon for the regulation of behaviors of biofilm formation and virulence. ${ }^{2-4}$ QS Comprises of chemical communication among bacteria involving formation, secretion, detection and reaction to molecules known as autoinducers (AI). Several serious infections are reported to be a result of biofilm formation and further it leads to chronic diseases. These Persistent infections is a challenge for public health on a global scale as it reduces the effectiveness of treatments and increases morbidity, mortality, and health care costs. ${ }^{5}$ Streptococcus mutans is an impotent pathogen and is a common cause of oral infections such as dental caries; $S$. mutans effectively utilizes dietary sucrose to synthesize large amounts of exo polysaccharides, which plays an important role in accumulation, adhesion, plaque matrix formation of microorganism, These processes in most cases lead to serious infections. The ability of micro-organism to from biofilm on host tissue surface is an important step in the development of infection. ${ }^{6}$ Due to poor hygienic condition and infection of pathogenic micro-organism creates difficulties and presently wide range of antibiotics are being used for treating infections but due to their adverse effect and antibiotic resistance is now paying attention towards natural biologically active herbal compounds as an alternative medicine. ${ }^{7,8}$ Natural compounds Curcumin commonly found in Curcuma longa it's a diarylheptanoid, the principal component of Curcuma longa. Negundoside it's a type of glycoside found in Vitex negundo are available plant species in India. It is a reputed medicinal herb and employed as a traditional cure in Asian system of medicine (Indian, Chinese and Malaysian) for variety of disease conditions. ${ }^{9-12}$ The objective of present study was to identify natural compounds like Curcumin and Negundoside for their potential against biofilm producing activity.

\section{Material and methods}

\section{Bacterial Isolation and Identification}

Streptococcus mutans were isolated from dental caries or plaque from patients of OPD's Peoples Dental Academy, Bhopal, M.P., India. These patients were of both sexes with the mean age of 20 years. The standard strain was S. mutans ATCC 25175. All samples were cultured on the media such as Brain Heart Infusion agar (BHI, Himedia Laboratories, India) in a $5 \% \mathrm{CO}_{2}$ enriched atmosphere and Mutant Sanguis Agar (MSA, Himedia Laboratories, India). The biochemical tests were done for their identification. Among 20 samples from patients having dental caries, 30 isolates were identified as $S$. mutans. 


\section{Preparation of natural compounds}

Natural compounds Curcumin and Negundoside were procured from Natural remedies India, were dissolved at a concentration 10mg/ $\mathrm{ml}$ in dimethylsulfoxide (DMSO, Mark).

\section{Screening of S. mutans for biofilm formation}

Microtiter plate method- Quantification of $S$. mutans isolates biofilm formation was carried out by using microtiter plate method. To assay biofilm formation of the $S$. mutans isolates, an overnight culture of each was grown in brain heart infusion broth (Himedia Laboratories, India) for $18-20 \mathrm{~h}$ at $37^{\circ} \mathrm{C}$. One $\mathrm{ml}$ of each overnight culture was transferred to $10 \mathrm{ml}$ of sterile BHI broth with the addition of $1 \%$ Sucrose for biofilm production. The suspensions were adjusted with the same BHI medium to 0.5 on the McFarland turbidity standard as measured by absorbance $(0.08-0.1$ at $625 \mathrm{~nm})$ in a spectrophotometer (Shimadzu, Australia), corresponding to approximately $10^{2} \mathrm{cfu} / \mathrm{ml}$. Then, from each culture, $250 \mu$ l volumes were transferred into wells of a microtiter plate (Himedia Laboratories, India). ${ }^{13}$ Blank wells contained broth, only. Plates were made in triplicate and incubated for $24 \mathrm{~h}$ at $37^{\circ} \mathrm{C}$. At the end of $24 \mathrm{~h}$, the planktonic suspension and nutrient solution were aspirated and each well was washed three times with $300 \mu$ of sterile physiological saline. The plates were strongly shaken in order to remove all non-adherent bacteria. The remaining attached bacteria were fixed with $250 \mu \mathrm{l}$ of $96 \%$ ethanol per well and, after $15 \mathrm{~min}$, plates were made empty and left to dry. Each well was then stained for 5 min with $200 \mu \mathrm{l}$ of $2 \%$ crystal violet (CV Gram stain, Merck, Germany). The stain was rinsed off by placing the plates under running tap water. After drying the stained plates, biofilms were visible as purple rings formed on the sides of each well. The quantitative analysis of biofilm formation was performed by adding $200 \mu 1$ of $33 \%$ (v/v) glacial acetic acid (Merck, Germany) per well. Then the optical density (OD) of the stain was measured at $492 \mathrm{~nm}$ by an ELISA reader (Lisa, Germany) as described previously. ${ }^{13}$ Biofilm formation was scored as follows: -, non-biofilm-forming ( $A 492 \leq 1)$; + , weak $(1 \leq A 492 \leq 2)$; ++, moderate $(2<A 492 \leq 3)$; +++ , strong $(A 492>3)$. Microtiter assay was performed in triplicate.

\section{Microscopic analysis by using the coverslip method}

Biofilm of $S$. mutans clinical isolates were grown as follows, individual sterile culture dishes were filled with $2.5 \mathrm{ml}$ of BHI broth with $1 \%$ sucrose and sterile $18 \mathrm{~mm}$ diameter glass microscope cover slip was added to each dish, and culture dishes were covered. Each sample was inoculated with defined volume of overnight culture. The dishes were incubated micro aerobically at $37^{\circ} \mathrm{C}$ for $48 \mathrm{hr}$. Glass cover slips containing attached biofilm were removed from dishes and rinsed briefly with PBS and stained with $0.5 \%$ crystal violet for $5 \mathrm{~min}$. Stained biofilm were observed microscopically. ${ }^{14}$

\section{Determination of minimum inhibitory concentration of curcumin and negundoside}

The minimum inhibitory concentration(MIC) of natural compounds Curcumin and Negundoside were evaluated on all the isolates by broth dilution methods. The natural compounds were dissolved in dimethyl sulfoxide (DMSO), initial concentration was $2 \mathrm{mg} / \mathrm{ml}$ to $0.0078 \mathrm{mg} /$ $\mathrm{ml}$. The initial test concentration was serially diluted two-fold. Each well was inoculated with $5 \mu \mathrm{L}$ of suspension containing $10^{8} \mathrm{cfu} / \mathrm{mL}$ of bacteria. The plates with bacteria were incubated for $24 \mathrm{~h}$ at $37^{\circ} \mathrm{C}$. After incubation, $5 \mu \mathrm{L}$ of tested broth was placed on the sterile BHI plates and incubated at respective temperature. The MIC for bacteria was determined as the lowest concentration of the extracts inhibiting the visual growth of the test cultures on the agar plate. Triplicates were maintained. ${ }^{15,23}$

\section{Biofilm inhibition assay in presence of curcumin and negundoside}

Only those isolates of S. mutans that were classified as strong biofilm producers were used in the biofilm inhibition assay. Test compounds were dissolved in DMSO $(10 \mathrm{mg} / \mathrm{ml})$, and two fold dilutions were made to result in a final concentration ranging from $2-0.0078 \mathrm{mg} / \mathrm{ml}$ in the wells after the addition of the freshly diluted brain heart infusion broth culture containing $10^{6} \mathrm{cfu}$ of the strong biofilm forming isolates per well. After incubation for $24 \mathrm{~h}$ at $37^{\circ} \mathrm{C}$, microtiter plate was washed, fixed and biofilms were stained and visualized as outlined above. The inhibitory effect of the plant compound on biofilm production was calculated by subtracting the media control. The biofilm inhibition concentration (BIC) is the concentration of the natural compound at which the biofilm formation was reduced to an absorbance $492<0.5$ OD. Each assay for BIC determination was performed in triplicate.

\section{Statistical analysis}

Calculations and statistics were performed using GraphPad 5.0 software (GraphPad Software Inc., La Jolla, CA). The results were analyzed using one-way analysis of variance (ANOVA). Significance was defined as $\mathrm{P}<0.05$. Results are presented as mean \pm the standard error of the mean (S.E.M.).

\section{Results}

\section{Screening of S. mutans for biofilm formation}

The $30 \mathrm{~S}$. mutans isolates were screened for biofilm formation by the microtiter plate method and the results are shown in Figure 1, all 30 isolates were classified by their biofilm forming potential as follows: 18 isolates were strong biofilm producers, 9 isolates were shown moderate producer and 3 isolates were shown poor/non biofilm producers. The $S$. mutans ATTCC was included as an assay control and found it to be a moderate biofilm producer. The observations confirm that the magnitude and intensity of biofilm formation of 18 isolates were significantly greater than those of the poor/nonbiofilm producer. The result of Figure 1 also shows the quantitative evaluation of identifying and demarcating strong biofilm producing $S$. mutans isolate from moderate and poor/nonbiofilm producing isolates. The biofilm forming potential of the strong producers at $\mathrm{OD}_{492}$ was greater than 1 , whereas non-biofilm producing isolates at $\mathrm{OD}_{492}{ }_{492}$ was less than 1. The isolate SM03 was identified as the strongest biofilm producer, whereas SM15 and SM27 were identified as moderate and poor/non biofilm producers respectively.

\section{Microscopic analysis of biofilm formation}

For visualization of biofilm formation by three categories of $S$. mutans isolates (Strong, Moderate, and poor/non biofilm producer), the microscopic slide assay was performed and the results are shown in figure 2. The biofilm formation was clearly visible for the strong biofilm producer SM03 followed by the moderate biofilm producer SM15 at $48 \mathrm{~h}$ and the poor/non biofilm producer SM27 did not form the biofilm even after $48 \mathrm{~h}$. The strong biofilm producer also showed strong adherence to the slide and thus one of the strongest biofilm producer SM03 isolate was selected for evaluating the MIC and BIC. 


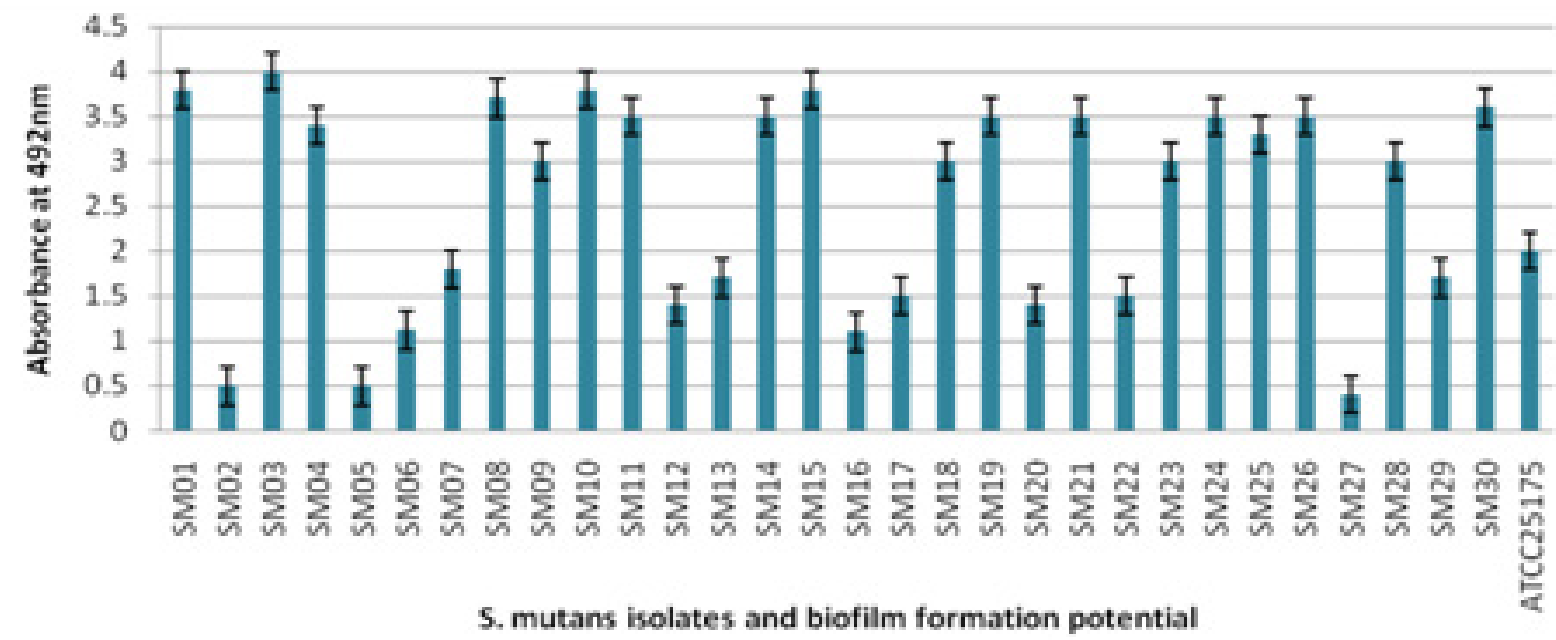

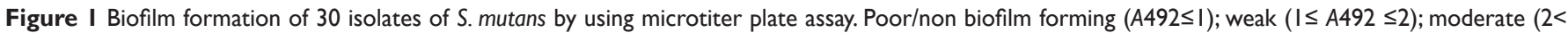
A492 $\leq 3)$; strong $(A 492>3)$.

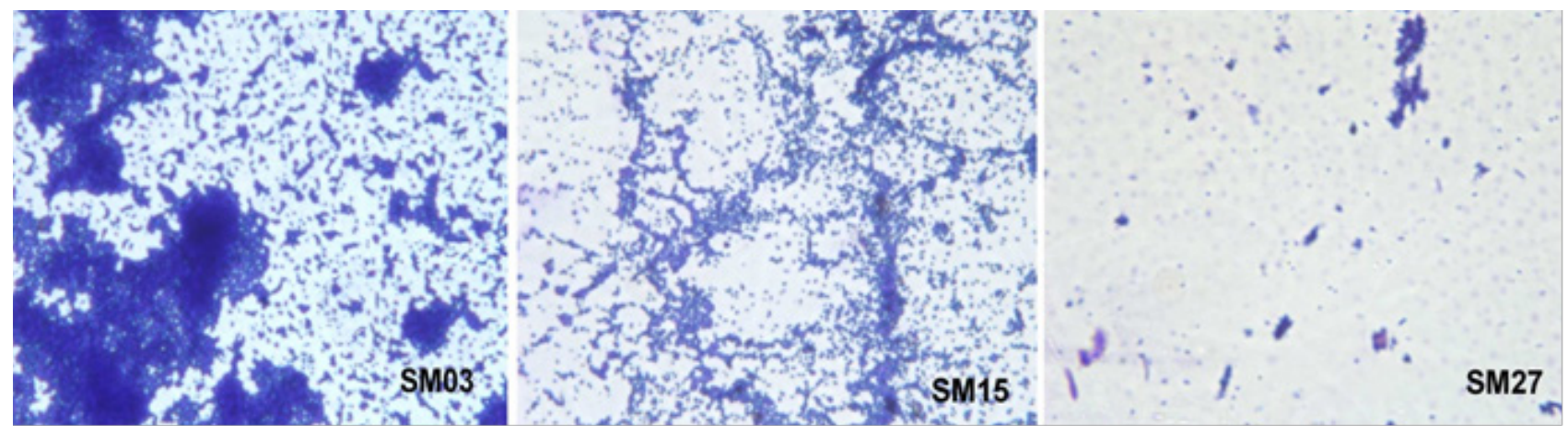

Figure 2 Microscopic visualization of Biofilm Production, where SM03 - Strong Biofilm Producer, SMI5 - Moderate and SM27 - Poor/non Biofilm Producer.

\section{Determination of MIC and BIC of curcumin and negundoside}

The results of Minimum Inhibitory Concentration (MIC) and Minimum Biofilm Inhibitory Concentration (MBIC) of the natural compounds analyzed for the strongest biofilm producer isolates SM03 are presented in Table 1. A significance difference in the MIC and BIC of the natural compounds was noted: the MIC of Curcumin are $0.63 \pm 0.02$ whereas that of Negundoside was $0.48 \pm 0.02$ (MIC Negundoside $<$ Curcumin). The Minimum Biofilm Inhibitory Concentration (MBIC) of Curcumin was $0.0570 \pm 0.03$, whereas that of Negundoside was 0.04170 .03 . These above results confirm that the Negundoside is the most potent antimicrobial and antibiofilm compound (Negundoside $>$ Curcumin).

\section{Biofilm inhibition of strong biofilm producers}

The results of Biofilm Inhibition by the natural compounds Curcumin and Negundoside in each strong biofilm forming S. mutans isolates are presented in Table 2. These results clearly confirms that the MBIC of Curcumin and Negundoside has reproducible biofilm inhibitory activity against each of strong biofilm producing isolates of $S$. mutans. The comparative effect of Minimum Biofilm Inhibition Concentration of Curcumin $0.0570 \pm 0.03$ and Negundoside 0.04170 .03 at $\mathrm{OD}_{492}<0.5$ shows the significant inhibition of biofilm. Therefore data of minimum biofilm inhibition concentration truly reflects Negundoside $>$ Curcumin compounds have potent ability to inhibit biofilm formation.

Table I The Comparative effect of two different natural compounds on growth inhibition versus biofilm inhibition. The values were determined for the high biofilm forming isolate SM03.Values are mean \pm S.E.M. of three replicates

\begin{tabular}{llll}
\hline S. No. & Compound & $\begin{array}{l}\text { Minimum inhibitory concentration (MIC) } \\
\mathbf{m g} / \mathbf{m L}\end{array}$ & Biofilm inhibition concentration (BIC) $\mathbf{m g} / \mathbf{m L}$ \\
\hline I & Curcumin & $0.63 \pm 0.02$ & $0.0570 \pm 0.03$ \\
2 & Negundoside & $0.48 \pm 0.02$ & $0.0417 \pm 0.03$
\end{tabular}

Comparative study of herbal compounds Curcumin and Negundoside on the biofilm producing property of Streptococcus mutans isolated from oral infection 
Table 2 Biofilm inhibition by the Curcumin and Negundoside in high biofilm forming isolates of S. mutans.Values are mean \pm S.E.M. of three replicates. (Statistically significant at $\mathrm{P}<0.05$ )

\begin{tabular}{|c|c|c|}
\hline \multirow{2}{*}{ Isolate No. } & \multicolumn{2}{|c|}{$\begin{array}{l}\text { Absorbance at } 492 \mathrm{~nm} \text { for Biofilm Inhibition in the } \\
\text { presence of natural compounds }\end{array}$} \\
\hline & Curcumin $0.0570 \mathrm{mg} / \mathrm{mL}$ & Negundoside $0.0417 \mathrm{mg} / \mathrm{mL}$ \\
\hline SMOI & $0.17 \pm 02$ & $0.13 \pm 02$ \\
\hline SM03 & $0.27 \pm 02$ & $0.22 \pm 02$ \\
\hline SM04 & $0.20 \pm 02$ & $0.18 \pm 02$ \\
\hline SM08 & $0.23 \pm 02$ & $0.20 \pm 02$ \\
\hline SM09 & $0.20 \pm 02$ & $0.23 \pm 02$ \\
\hline SMIO & $0.24 \pm 02$ & $0.17 \pm 02$ \\
\hline SMII & $0.19 \pm 02$ & $0.13 \pm 02$ \\
\hline SMI4 & $0.21 \pm 02$ & $0.11 \pm 02$ \\
\hline SMI5 & $0.23 \pm 02$ & $0.17 \pm 02$ \\
\hline SMI8 & $0.20 \pm 02$ & $0.12 \pm 02$ \\
\hline SMI9 & $0.19 \pm 02$ & $0.21 \pm 02$ \\
\hline SM2I & $0.22 \pm 02$ & $0.15 \pm 02$ \\
\hline SM23 & $0.21 \pm 02$ & $0.12 \pm 02$ \\
\hline SM24 & $0.18 \pm 02$ & $0.13 \pm 02$ \\
\hline SM25 & $0.20 \pm 02$ & $0.18 \pm 02$ \\
\hline SM26 & $0.17 \pm 02$ & $0.11 \pm 02$ \\
\hline SM28 & $0.22 \pm 02$ & $0.14 \pm 02$ \\
\hline SM30 & $0.21 \pm 02$ & $0.16 \pm 02$ \\
\hline $\begin{array}{l}\text { Control ATTCC } \\
25175\end{array}$ & $0.22 \pm 02$ & $0.23 \pm 02$ \\
\hline
\end{tabular}

\section{Discussion}

S. mutans have significant role in dental infection by effectively utilization of sugars and synthesize large amounts of exo polysaccharides and important role in adhesion of bacteria and accumulation of biofilm. Thus organized structure and mechanisms of biofilm are responsible for the emergence of drug resistance in bacteria. ${ }^{16}$ The present allopathic formulations which is used in oral care contain antibiotics, antimicrobial agents, surfactants and alcohol, are not efficient in eradicating oral pathogens completely; on the contrary they were found to be cytotoxic. ${ }^{17,18}$ So the plants derived products are of choice against oral pathogens. Hence, in this study natural compounds Curcumin commonly found in Curcuma longa it's a diarylheptanoid, and principal component of Curcuma longa, Negundoside it's a type of glycoside found in Vitex negundo are available plant species in India, chosen for the biofilm inhibition study. ${ }^{19,22}$

The objective of this study was to evaluate natural compounds biofilm inhibition of clinical isolates of $S$. mutans. Towards this, 30 clinical isolates were screened, out of which 18 isolates strong biofilm producers were identified (Figure 1) the effects of Curcumin and Negundoside were tested for their inhibitory effects on bacterial growth and effect on the biofilm formation in a representative strongest biofilm forming isolate SM03 (Table 1) to determine the minimal concentration required for biofilm inhibition. Further this concentration was used against each of the high biofilm producer isolates.

The comparative effects of Curcumin and Negundoside compounds on growth inhibition versus biofilm inhibition are summarized in Table 1. The biofilm inhibition concentration is significantly lower than the concentration required for inhibition of bacterial growth. erefore data of minimum biofilm inhibition concentration truly reflect Negundoside $>$ Curcumin compounds have potent ability to inhibit biofilm formation. There are reason behind this, compound tested may have inhibited receptors and molecules involved in the quorum sensing pathway which is required for biofilm formation. ${ }^{20-23}$

Effects of Curcumin and Negundoside compounds on biofilm inhibition at minimum concentration, data suggested that MBIC value also shows the effective potential to inhibit the biofilm formation by the strong biofilm producer isolates. Absorbance at $492 \mathrm{~nm}$ for Biofilm Inhibition in the presence of natural compounds suggested that significant potential of biofilm inhibition of Negundoside $>$ Curcumin (Table 2). 


\section{Conclusion}

Comprehensive analysis of the effect of Curcumin and Negundoside compounds on strong biofilm forming isolates of $S$. mutans. The results show that both the natural compound has potent biofilm inhibition. It would be interesting to find out the mode of action or cell to cell inhibition action to inhibit biofilm formation. These compounds would be very useful in controlling biofilm forming infection of $S$. mutans.

\section{Acknowledgments}

The authors are grateful to the Department of Microbiology, Barkatullah University, Bhopal M.P. for laboratory support.

\section{Conflicts of interest}

Author declares there are no conflicts of interest.

\section{Funding}

None.

\section{References}

1. Sandasi M, Leonard CM, Viljoen AM. The in vitro antibiofilm activity of selected culinary herbs and medicinal plants against Listeria monocytogenes. Lett In App Microbiol. 2010;50(1):30-35.

2. Waters CM, Bassler BL. Quorum sensing:Cell-to-cell communication in bacteria. Annu Rev Cell Dev Biol. 2005;21:319-346.

3. Reading NC, Sperandio V. Quorum sensing: The many languages of bacteria. FEMS Microbiol Lett. 2006;254(1):1-11.

4. Taga ME. Bacterial signal destruction. ACS Chem Biol. 2007;2(2):8992

5. Filoche S, Wong L, Sissons CH. Oral Biofilms: Emerging Concepts and Microbial Ecology. J Dent Res. 2010;89(1):8-18.

6. Florence G, Daniel G and Denis M. Oral microbial heat-shock proteins and their potential contributions to infections. Crit Rev oral Biol Med. 2003;14(6):399-412.

7. Al-Haroni M, Skaug N. Incidence of antibiotic prescribing in dental practice in Norway and its contribution to national consumption. $J$ Antimicrob Chemother. 2007;59(6):1161-1166.

8. Raghunath D. Emerging antibiotic resistance in bacteria with special reference to India. J Biosci. 2008;33(4):593-603.
9. Quality Standards of Indian Medicinal Plants. New Delhi, India: Indian Council of Medicinal Research (ICMR); 2008.

10. The Ayurvedic Pharmacopoeia of India; Part - I Volume - II Government of India. Department of Ayush, New Delhi, India: Government of India Ministry of Health And Family Welfare: 2008. p. 1-177.

11. Indian Herbal Pharmacopeia. Mumbai, India: Indian Drug Manufacturers Association; 2002. p. 206-313.

12. Tasduq SA, Kaiser PJ, Gupta BD, et al. Negundoside, an iridiod glycoside from leaves of Vitex negundo, protects human liver cells against calcium-mediated toxicity induced by carbon tetrachloride. World J Gastroenterol. 2008;14(23):3693-3709.

13. O'Toole GA, Kolter R. Initiation of biofilm formation in Pseudomonas fluorescens. Mol Microbiol. 1998;28(3):449-461.

14. Merritt JH, Kadouri DE, O'Toole GA. Growing and analyzing static biofilms. Curr Protoc Microbiol. 2005; Chapter 1:Unit 1B.1.

15. Dwivedi D, Patidar RK, Singh V. Antioxidant and antibacterial potential of Murraya konigii against human cariogenic pathogens. Int J Pharm Sci Res. 2012;3(9):3399-3406.

16. Lewis K. Riddle of biofilm resistance. Antimicrob Agents Chemother. 2001:45(4):999-1007.

17. Flemingson, Pamela E, Ambalavanan, et al. Effect of three commercial mouth rinses on cultured human gingival fibroblast:An in vitro study. Indian J Dent Res. 2008;19(1):29-35.

18. Costerton JW, Stewart PS and Greenberg EP. Bacterial biofilms:a common cause of persistent infections. Science. 1999;284(5418):13181322 .

19. Pilar T, Angel GI, Francisco TB, et al. Inhibition by chestnut honey of N-acyl-L-homoserine lactones and biofilm formation in Erwinia carotovora, Yersinia enterocolitica, and Aeromonas hydrophila. J Agric Food Chem. 2009;57(23):11186-11193.

20. Packiavathy IASV, Agilandeswari P, et al. Antiquorum sensing and antibiofilm potential of Capparis spinosa. Arch Med Res. 2011;42(8):658-668

21. Chenia HY. Anti-quorum sensing potential of crude Kigelia Africana fruit extracts. Sensors. 2013;13(3):2802-2817.

22. Aggarwal BB, Sundaram C, Malani N, et al. Curcumin: the Indian solid gold. Adv Exp Med Biol. 2007;595:1-75.

23. Al-Dhabi NA, Balachandran C, Raj MK, et al. Antimicrobial, antimycobacterial and antibiofilm properties of Couroupita guianensis Aubl. fruit extract. BMC Complem Altern Med. 2012;12:242. 